\title{
EL DEFENSOR DEL PUEBLO COMO INSTRUMENTO PARA AFIANZAR EL DERECHO Y LA DEMOCRACIA. ALGUNAS REFLEXIONES
}

\author{
Manuel Vidaurri Aréchiga*
}

\begin{abstract}
Sumario:
1.- Introducción. 2.- Repaso breve sobre funciones y atribuciones del Defensor del Pueblo. 3.Problemática institucional. 4.- Algunas propuestas de mejora. 5.- Los Principios de París y su impacto en las instituciones defensoriales. 6.- Una reforma constitucional paradigmática. 7.- El Defensor del Pueblo y su aportación a la democracia.
\end{abstract}

Resumen: Este artículo ofrece algunas opiniones en torno al papel de las instituciones protectoras de derechos humanos, llamadas en algunos países Ombudsman o Defensorías del Pueblo, en relación con la democracia y la vigencia del derecho. Se hace una sucinta revisión de las funciones asignadas y problemática institucional para ofrecer luego algunas reflexiones genéricas.

Palabras clave: Obdusman, Derechos Humanos. Democracia.

\begin{abstract}
This article offers some views on the role of institutions protecting human rights in some countries calls Ombudsmen in relation to democracy and the observance of law. It is a brief review of the functions and institutional problems to offer some thoughts after generic

Palabras clave: Defensor del Pueblo. Derechos Humanos. Democracia.
\end{abstract}

Keywords: Ombudsman. Human Rights. Democracy.

\section{INTRODUCCIÓN}

Las que siguen son, más que ideas acabadas o definitivas, intentos de reflexiones que se hacen en voz alta en torno al gran tema que nos reúne ahora.

Como es comprensible, la temática elegida dista mucho de ser de fácil acometida. Sobre todo teniendo en cuenta que cada uno de los elementos que se implican -Defensor del Pueblo, Democracia, Derecho- constituyen, por sí mismos, conceptos complejos no sólo desde el punto de vista teórico, tanto como desde la perspectiva de la praxis social y cultural. Preguntarnos hoy si la figura del Defensor del Pueblo es, en realidad, un instrumento para afianzar el Derecho y la Democracia, no deja de ser un atractivo ejercicio intelectual y científico.

* Profesor- investigador del Departamento de Derecho de la División de Derecho, Política y Gobierno de la Universidad de Guanajuato. 
Habrá quienes, por supuesto, consideren que estas instituciones no estén en condiciones de contribuir en algo a la colmación de tan complejas expectativas sociales como vivir en un Estado Democrático y de Derecho. Probablemente en algún aspecto tengan razón. Sin embargo, en adelanto de ciertas voces críticas, preferimos ocuparnos de aquello que sí pueden -o deben- hacer los referidos organismos en la búsqueda de ese loable propósito de afianzamiento del Derecho y la Democracia.

\section{REPASO BREVE SOBRE FUNCIONES $Y$ ATRIBUCIONES DEL DEFENSOR DEL PUEBLO}

Conviene hacer un breve recordatorio sobre las funciones generalmente asignadas al Ombudsman o Defensor del Pueblo. ${ }^{1}$ Al margen de las obvias diferencias legales propias de cada organismo nacional, siguiendo la autorizada voz de Jorge Santiestevan de Noriega, ${ }^{2}$ quien fuera primer Defensor del Pueblo del Perú, entre las funciones o atribuciones más significativas de estos organismos se encuentran estas:

$\checkmark$ Defensa y protección de los derechos humanos.

$\checkmark$ Investigación y elaboración de resoluciones respecto de violaciones a derechos humanos.

$\checkmark$ Denuncia a la autoridad correspondiente por violaciones a derechos humanos.

$\checkmark$ Educación y promoción en y de los derechos humanos.

$\checkmark$ La función de mediación.

$\checkmark$ Potestad de iniciativa legislativa.

$\checkmark$ Interponer acciones de inconstitucionalidad.

El catálogo de atribuciones puede ser más o menos extenso, pero en casi todas las legislaciones se consideran las ya apuntadas mismas que, por cierto, no son pocas. Así, pues, tenemos que defender, proteger, denunciar, educar y mediar son verbos rectores de las eventuales acciones a desplegar por el Ombudsman, con base en las facultades otorgadas por la ley.

En tal sentido, si se trata de afianzar la prevalencia del Derecho (el interno y el propio de los acuerdos y tratados internacionales suscritos por el

\footnotetext{
${ }^{1}$ Utilizaremos indistintamente el concepto Defensor del Pueblo u Ombudsman, en virtud de que así se les identifica de manera generalizada.

${ }^{2}$ Santiestevan de Noriega, Jorge, El Defensor del Pueblo en Iberoamérica, Perú, 2002, pág. 56 y ss.
} 
país), la acción defensora y garantísta del Ombudsman es una acción legalmente dispuesta, y en ocasiones, tal mandato deriva de la máxima norma jurídica de un país: la Constitución.

La denuncia ante la autoridad correspondiente, en caso de violaciones a derechos humanos que constituyan delitos o faltas de otra naturaleza jurídica, también es una vía para el restablecer el orden legal vulnerado.

Más complicado si se quiere, será el fortalecimiento de la democracia, la que no depende exclusivamente de estos organismos sino de muchos otros factores entre los que se encuentran, obviamente, los políticos, económicos y sociales. No obstante, las acciones educativas en y de promoción de los derechos humanos son el ámbito de oportunidad más notable desde el que, en efecto, es perfectamente posible contribuir al logro de este propósito. Ana María Rodino, reconocida experta en la materia integrante de la Unidad Pedagógica del entrañable Instituto Interamericano de Derechos Humanos dice algo que viene a cuento: "la Educación en Derechos Humanos enseña la importancia de conocer, valorar, y respetar derechos de las personas como exigencia de su condición de seres humanos y como pautas de convivencia social inclusiva, justa, pacifica y solidaria". ${ }^{3}$

La cultura de paz, que se logra muchas veces a través de la utilización de los medios alternativos de resolución de controversias, no es tarea menor, y antes bien, fortalece e impulsa la construcción de sociedades que actúan inteligente y racionalmente ante la existencia de un conflicto solucionable con base en la buena voluntad e interés de las partes involucradas.

La capacidad de iniciar leyes o la posibilidad de interponer acciones de inconstitucionalidad, resultan fortalezas de indudable poder transformador del orden jurídico.

Ciertamente, desde la perspectiva meramente formal, es decir legal, estas atribuciones dan la impresión de que la tarea del Ombudsman es más que sencilla. Sin embargo, otra es, en efecto, la realidad.

\section{PROBLEMÁTICA INSTITUCIONAL}

Un diagnóstico general sobre las Defensorías de Iberoamérica, elaborado en el marco del Programa Regional de Apoyo a las Defensorías del Pue-

\footnotetext{
${ }^{3}$ Véase su artículo "Educación en Derechos Humanos y Democracia", en el libro La promoción y protección de los derechos humanos hoy, Memoria de los Seminarios Interinstitucionales sobre la Promoción y Protección de los Derechos Humanos, México, 2006, pág. 174.
} 
blo de Iberoamérica (PRADPI, por sus siglas) ${ }^{4}$ dejo en claro algunos de los problemas por los que atraviesan estas instituciones. Muchos de estos aspectos repercuten negativamente en la acción del Ombudsman, afectando seriamente en algunos casos ese pretendido afianzamiento del Derecho y fortalecimiento de la Democracia.

No será esta la ocasión en la que nos detengamos a comentar cada uno de los puntos mencionados en el Diagnóstico en cuestión. En todo caso, seleccionamos algunas de las problemáticas detectadas:

$>$ El desempeño de algunos Defensores ha hecho que se transmita una idea de provisionalidad y de politización de las Defensorías. En ocasiones, se da la cuestión de que el Defensor se ha convertido en el centro del debate político partidario, llegándose en algunos casos a exigir su dimisión por presión política y social. El efecto producido es, lamentablemente, el descrédito de la institución.

$>$ La politización en la elección del titular de la Defensoría, dándose el reprobable hecho de que, por acuerdos políticos sostenidos por los partidos políticos, no siempre se elige a la persona más adecuada o idónea para el ejercicio del cargo.

$>$ La utilización del cargo de Defensor para que el titular del mismo se proyecte a otros cargos públicos. Se advierte que, en este supuesto, la Defensoría pasa de ser un instrumento ciudadano, para convertirse en un instrumento del poder.

$>$ Se reconoce en el Diagnostico que el presupuesto asignado para el funcionamiento del organismo es, generalmente, escaso, amén de que deben realizarse gestiones "políticas" a fin de obtener los recursos indispensables.

$>$ El perfil del Defensor es fundamental, ya que su liderazgo incide positivamente en el mejor desempeño de las tareas y funciones propias del organismo. Según el Diagnostico, se conocieron casos de que el Defensor "suele ser una persona sensible frente al desvalimiento de una parte mayoritaria de la sociedad y están convencidos de que la organización es un instrumento para defender los derechos humanos de la población. Suelen hacer de la prudencia una norma, y se congratulan del buen entendimiento con todos los poderes del Estado".

\footnotetext{
${ }^{4}$ Contenido en el Manual de Buenas Prácticas Institucionales de las Defensorías de Iberoamérica, Centro de Iniciativas de Cooperación al Desarrollo, Universidad de Alcalá, Madrid, 2004, páginas 211 y siguientes.
} 
$>$ En suma: quedó evidenciada la gran vulnerabilidad de estas instituciones ante las presiones políticas ejercidas por los partidos que gobiernan los distintos partidos de la región.

Por lo que se puede apreciar, es posible que las leyes que rigen la vida y acciones de estos organismos sean el paradigma de la perfección en materia de defensa y promoción de los derechos humanos. Por desgracia, los datos reportados antes, revelan esa situación real y preocupante que se opone, en mayor o menor medida, al logro de la más importante misión globalmente considerada del Defensor del Pueblo: garantizar a las personas todos sus derechos, así como el libre ejercicio de las prerrogativas que la ley les reconoce.

\section{ALGUNAS PROPUESTAS DE MEJORA}

El Diagnóstico ofrece propuestas destinadas a la mejora del organismo defensorial. Entre otras ponen las siguientes:

$\checkmark$ Que las Defensorías alcancen rango constitucional para cimentar su autonomía y evitar con ello las presiones de los poderes a los que tienen que controlar. Y textualmente señala: "La Defensoría es un instrumento único, de primerísimo orden para alcanzar una república justa y democrática, y para su plena capacidad de desenvolvimiento en el cumplimiento de sus fines no debe retrasarse el dotarla de mayor legitimidad, que es la legitimidad constitucional, donde no se haya alcanzado todavía".

$\checkmark$ Que la labor de los Defensores ante los poderes del Estado debe estar presidida por la colaboración, pero sin merma de su capacidad para el desempeño adecuado de su misión.

$\checkmark$ Es imprescindible que el titular de la Institución, en el ejercicio de su función, actúe con independencia respecto de partidos políticos; incluso aunque en el pasado haya sido miembro de alguno de ellos. De otra forma, la legitimidad y la credibilidad institucional se verán minadas ante la población.

$\checkmark$ Una propuesta más, que considero importantísima: "Las Defensorías del Pueblo podrían intervenir proponiendo mejoras en el ordenamiento jurídico en aquellos casos en los que, además de estar implicados los derechos de los ciudadanos, se trate de aspectos que tengan que ver con la lucha contra la corrupción y a favor de la transparencia y el comportamiento ético de los representantes y servidores públicos". ${ }^{6}$

\footnotetext{
${ }^{5}$ Diagnóstico... Op. cit., p. 228.

${ }^{6}$ Diagnóstico... Op. cit., p. 230.
} 
$\checkmark$ El organismo debe cumplir su misión fundamental: atender a la población vulnerada y vulnerable. Procediendo de esta manera, se impone en la cultura política de los Estados la necesaria presencia y adecuado desempeño laboral de las Defensorías como garantía del funcionamiento democrático de los poderes.

$\checkmark$ Propuesta clave, sin duda, la de establecer una estrecha relación de coordinación entre las Defensorías y las organizaciones de la sociedad, con el fin de promocionar, defender y educar en materia de derechos humanos a la población en su conjunto.

En este rapidísimo recorrido por las funciones, situación problemática y algunas propuestas de mejora para la actividad defensorial, podemos constatar que son más las ventajas que las desventajas, pues se cuentan con estructuras legales que potencian el ser y quehacer del Ombudsman, lo que significa que desde el plano de lo estrictamente formal estos organismos están en condiciones de cumplir cabalmente con su misión institucional.

\section{LOS PRINCIPIOS DE PARÍS Y SU IMPACTO EN LAS INSTITUCIONES DEFENSORIALES}

Los denominados Principios de París, fueron aprobados por la Asamblea General de las Naciones Unidas en Diciembre de 1993 (Resolución 48/ 134). Como muchos saben, estos Principios se refieren al estatus, competencias, atribuciones y funciones de las instituciones nacionales de protección y promoción de los derechos humanos. Las principales características de estos Principios son:

$\checkmark$ Un fundamento constitucional y un estatuto jurídico propios

$\checkmark$ Un mandato tan amplio como sea posible

$\checkmark$ Autonomía de gestión, con reglamentos especificados por la ley

$\checkmark$ Una composición pluralista y representativa

$\checkmark$ Independencia del Poder Ejecutivo

$\checkmark$ Recursos económicos adecuados.

Como un complemento a estas características, son interesantes las propuestas realizadas por una de las organizaciones civiles más influyentes internacionalmente como lo es Human Rihgts Watch, contenidas en una publicación de 2001 titulada Protectores or Pretenders. En tal texto sugieren que las responsabilidades de las instituciones nacionales incluyen: 
$\checkmark$ Reportar y elaborar recomendaciones al gobierno en materia de derechos humanos.

$\checkmark$ Promover la conformidad de las leyes y prácticas nacionales con los estándares internacionales de derechos humanos.

$\checkmark$ Cooperar con los organismos de derechos humanos nacionales, regionales y de Naciones Unidas.

Sin dejar de reconocer la importancia de los Principios de París, cabe hacer notar que se trata de principios mínimos, lo que no impide que los Estados promuevan aquellos otros que, finalmente, consoliden una cultura social en torno de la vigencia y vivencia de los derechos humanos.

Lo interesante de estos principios, es que, al referirlos al tema de este Seminario, cobran una actualidad indiscutible. Sobre todo porque revitalizan el debate en torno al desarrollo institucional experimentado por los organismos desde la fecha de expedición de los Principios en 1993 al momento actual. Del mismo modo, indicar que el Defensor del Pueblo es un instrumento de fortalecimiento del Derecho y la Democracia nos obliga, inexorablemente, a indagar sobre las fortalezas y debilidades institucionales propias del organismo.

Jody Kollapen, quien para el 2004 fungía como Presidente de la Comisión Sudafricana de Derechos Humanos, al ocuparse de los citados Principios de Paris, ${ }^{7}$ recalcó la importancia de los instrumentos internacionales en tanto constituyen el marco normativo referencial para el trabajo de los organismos protectores de derechos humanos, pero asume, con razón, que los instrumentos por sí mismos no pueden cambiar una realidad social, política y económica.

El mismo representante sudafricano describió los requerimientos mínimos para el despliegue de un ambiente favorable a los derechos humanos. Entre estos requerimientos señalo: una democracia funcional con separación de poderes, un sistema de administración de justicia independiente, libertad de prensa, una activa sociedad civil y un marco legal o constitucional que procure el cumplimiento de las obligaciones nacionales e internacionales de los Estados en materia de derechos humanos. En tal modelo, el deber de promover e impulsar los derechos humanos recayó, principalmente en el Estados, y los tribunales por su parte, intervinieron en aquellos casos en lo que la acción estatal violentaba o amenazaba derechos fundamentales.

${ }^{7}$ Kollapen, Jody, "El papel y el lugar de las instituciones nacionales en el avance de la democracia y de los derechos humanos", en Retos actuales de las instituciones nacionales de protección y promoción de los derechos humanos. A diez años de los Principios de París, Comisión Nacional de los Derechos Humanos, México, 2004, p. 20. 
El mismo Kollapen reconoce que este sistema funcionó con cierto éxito en muchos aspectos, pero en muchos otros se dieron defectos importantes. "La realidad fue que -escribe el autor citado- inevitablemente el Estado era responsable de la mayoría de las violaciones a los derechos humanos. Esto no es insólito: si consideramos la magnitud del poder que un Estado tiene, incluso en las democracias más avanzadas, es inevitable la posibilidad de que el ejercicio de tal poder termine vulnerando los derechos humanos". ${ }^{8}$

En este repaso de la situación, no olvida mencionar la complejidad del acceso a la justicia para las personas pobres, las engorrosas reglas procesales así como otros temas propios de las instancias de procuración y administración de justicia, los que, por otro lado, no son los espacios institucionales más apropiados para tratar cuestiones relacionadas con derechos humanos.

Así pues, mientras por un lado, el Estado mediante el ejercicio del poder lamentablemente lesiona derechos humanos y, por otro, los tribunales no son considerados los mejores espacios para el análisis de los mismos, el escenario está dispuesto para la entrada en acción de los organismos de protección y defensa de derechos humanos, que es conceptualizado por Kollapen con estas palabras:

“Una institución nacional que, aunque reconoce los defectos y limitaciones de las disposiciones institucionales existentes, busca funcionar con creatividad e innovación; que tiene una capacidad proactiva y reactiva; que desarrolla una identidad distinta de la del Estado y de la sociedad civil, pero no tan alejada de ellos como para trabajar aisladamente; que tiene la independencia para determinar y definir su propio mandato; que es capaz de responder a los asuntos e intereses nacionales, regionales y locales sobre derechos humanos; que trabaja con el gobierno, la sociedad civil, la iniciativa privada y demás sectores, y que aunado a todo lo anterior se convierte en el centro del discurso y de la actividad sobre los derechos humanos del país".

Queda pues, descrito, el modelo ideal de Defensor del Pueblo, cuyas intervenciones y capacidad proactiva y reactiva hacen posible, según Kollapen, el avance de los derechos humanos dentro de una sociedad y fortaleciendo así la democracia y el proceso democrático. ${ }^{9}$

\footnotetext{
${ }^{8}$ Kollapen, op. cit., p. 20-21.

${ }^{9}$ Kollapen, op. cit., p. 26.
} 


\section{UNA REFORMA CONSTITUCIONAL PARADIGMÁTICA}

De los revisados Principios de París se alcanza a desprender un aspecto de vital importancia: el marco jurídico competencial del Defensor del Pueblo. Por supuesto, ese andamiaje jurídico puede y debe mejorar y ampliarse, hasta contar con las mejores herramientas jurídicas que permitan la más amplia protección de las personas.

Un ejemplo, relativamente reciente experimentado en la República Mexicana, fue la modificación al texto constitucional por la vía de la adición hecha al artículo 105, apartado II, inciso g), que faculta a los organismos defensores de derechos humanos para ejercitar las acciones de inconstitucionalidad.10 Textualmente el contenido del texto legal es el siguiente:

Artículo 105.- La Suprema Corte de Justicia de la Nación conocerá, en los términos que señale la ley reglamentaria, de los asuntos siguientes:

I.- $\ldots$.

II.- De las acciones de inconstitucionalidad que tengan por objeto plantear la posible contradicción entre una norma de carácter general y esta Constitución.

Las acciones de inconstitucionalidad podrán ejercitarse, dentro de los treinta días naturales siguientes a la fecha de publicación de la norma, por:

Incisos de la a) alf)

g) La Comisión Nacional de los Derechos Humanos, en contra de leyes de carácter federal, estatal y del Distrito Federal, así como de tratados internacionales celebrados por el Ejecutivo Federal y aprobados por el Senado de la República, que vulneren los derechos humanos consagrados en esta Constitución. Asimismo los organismos de protección de los derechos humanos equivalentes en los estados de la República, en contra de leyes expedidas por las legislaturas locales y la Comisión de Derechos Humanos del Distrito Federal, en contra de leyes emitidas por la Asamblea Legislativa del Distrito Federal.

Hasta antes de la reforma citada, los organismos protectores de derechos humanos carecían de legitimación activa para promover acciones de inconstitucionalidad. En su momento, señalamos que esta reforma creaba el que denominamos Derecho a la constitucionalidad, ${ }^{10}$ entendido como aquel que tienen todas y todos los habitantes de un territorio determinado en el sentido de esperar y recibir de las autoridades gubernamentales la realiza-

${ }^{10}$ El Decreto de reforma constitucional puede ver en el Diario Oficial de la Federación, del 14 de septiembre de 2006 . 
ción de actos de poder institucionales apegados al marco constitucional. También apuntamos que:

"La potencia de esta nueva facultad conferida -a lo mejor podríamos decir, reconocida- a los organismos protectores de derechos humanos, les dota de una herramienta muy poderosa a la hora de impulsar la efectividad de los derechos humanos (....) los actos legislativos, es decir, la formulación de leyes, ahora no solo serán observados y evaluados, sino que, en cierta forma, también serán "visados" por los organismos, sobre todo en lo concerniente a los tratados internacionales que versan sobre derechos humanos y su posible o real contradicción con la norma constitucional". ${ }^{11}$

Quisimos traer este ejemplo a cuenta porque, como puso de relieve Emilio Álvarez Icaza: ${ }^{12}$

“ La reforma al precepto constitucional referido constituye una respuesta oportuna a la solicitud que hicieran diversas organizaciones sociales y civiles de derechos humanos, tanto del Comité de Enlace para el Acuerdo de Cooperación Técnica en Materia de Derechos Humanos que suscribieron el Gobierno Federal y la Oficina del Alto Comisionado de las Naciones Unidas para los Derechos Humanos, como de la Comisión Política en Materia de Derechos Humanos, para el reconocimiento, como facultad de los Organismos Públicos de Derechos Humanos".

Llama la atención que este proceso de reforma constitucional haya surgido de inquietudes ciudadanas dirigidas, como es evidente, al fortalecimiento de los organismos defensores de derechos humanos, lo que obliga a reconocer nuevamente, que las organizaciones sociales contribuyen y mucho en los procesos de democratización. La alianza entre defensorías y grupos de la sociedad civil es, pues, virtuosa por conveniente.

Lo peor que puede sucederle al pueblo es que su Defensoría se autolimite o se retraiga de su responsabilidad hasta el punto de desaparecer del panorama. Las defensorías deben ser "un referente indispensable en la defensa de los derechos humanos, por su presencia activa permanente en los conflictos sociales que vayan surgiendo"; 13 corresponde a estos organismos encabezar la

\footnotetext{
${ }^{11}$ Vidaurri Aréchiga, Manuel, “El derecho a la constitucionalidad: los organismos públicos de derechos humanos y la acción de inconstitucionalidad", en revista Investigaciones Jurídicas, Facultad de Derecho de la Universidad de Guanajuato, Enero-Junio, número 82, México, 2009, p. 403.

${ }^{12}$ En "La autonomía de los organismos públicos y la jurisdiccionalidad de los derechos humanos en un Estado democrático de Derecho", publicado en Cinco Temas Fundamentales de Derechos Humanos, Edición de la Procuraduría de los Derechos Humanos del Estado de Guanajuato, México, 2007, p. 36.
} 
indignación y malestar social, impulsando las mejores causas con los mejores argumentos. Esta labor, por lo demás, requiere "firmeza, sensibilidad, discreción y capacidad de diálogo". ${ }^{14}$

El rol defensorial en favor de la cultura de y por la legalidad ha sido apenas pergeñado en estas páginas. Aunque, en apretada síntesis, podemos decir que promover e impulsar la cultura de y por la legalidad es una de las razones fundamentales e insoslayables para la existencia del Defensor del Pueblo: es parte de su tarea, parte de su responsabilidad y parte, incluso, de su legado institucional.

\section{EL DEFENSOR DEL PUEBLO Y SU APORTACIÓN A LA DEMOCRACIA}

En el marco del VII Congreso de la Federación Iberoamericana del Ombudsman, celebrado en noviembre del 2002 en Lisboa, Diogo Freitas do Amaral (Ex Ministro de Relaciones Exteriores de Portugal, entre otros destacados cargos) pronunció la conferencia magistral titulada: "Límites jurídicos, políticos y éticos en la actuación del Ombudsman".${ }^{15}$ En alguna parte de su conferencia señaló enfáticamente:

“Si los tres poderes inmortalizados por Montesquieu legislan, ejecutan y juzgan, el Ombudsman fiscaliza y controla la actuación administrativa de esos tres poderes, con plena independencia e imparcialidad, a fin de prevenir o reparar injusticias. Está fuera de los poderes Legislativo, Ejecutivo y Judicial, pero actúa sobre todos ellos, es un destacado y eminente órgano de garantía de los derechos fundamentales del individuo frente al Estado. Así sea que se le denomine Ombudsman, Provedor de Justicia, Defensor del Ciudadano, Mediador, o cualquier otro nombre este organismo es, en realidad, el protector institucional del ciudadano y de sus derechos fundamentales frente al poder. Es, pues, otro de los pilares básicos en los que se fundamenta el Estado democrático.

Inicialmente esos pilares eran tres; pero a partir del primer Ombudsman creado en Suecia en 1809, y rápidamente generalizado en el resto del mundo durante el siglo XX, esos pilares pasaron a ser cuatro.

${ }^{13}$ Así dicho en el Diagnóstico, op. cit., p. 212.

${ }^{14}$ Diagnóstico, op. cit., p. 213.

${ }^{15}$ Misma que apareció publicada en Democracia e Direitos Humanos no Século XXI, Lisboa, 2003. Hay traducción al español por Vidaurri Aréchiga, Manuel, en Revista Investigaciones Jurídicas, Facultad de Derecho de la Universidad de Guanajuato, número 82, México, Enero-Junio 2007. 
En mi opinión -dice Freitas do Amaral- el Ombudsman constituye el cuarto poder del Estado. Sé que esta designación suele asignárseles a los medios de comunicación, erradamente según pensamos, porque estos no son un poder del Estado sino un poder fáctico de la sociedad civil. Si Montesquieu volviera a vivir, con certeza no vacilaría, con todo su realismo y poder de observación, en proclamar en una nueva edición de su Esprit des lois que en la actualidad son cuatro los poderes del Estado".

En adición a lo dicho por el jurista y político portugués, podemos agregar que, en efecto, el Ombudsman o Defensor del Pueblo bien puede ser considerado como el titular de ese cuarto poder del Estado, pero no de cualquier Estado. Instituciones como estas difícilmente tienen cabida o recepción en Estados no democráticos. Luego, al ser institutos claramente democráticos y, en tal virtud, por congruencia política, les corresponde proteger e impulsar las características que definen al Estado democrático: la división de poderes, la alternancia en el poder, el pluripartidismo, el imperio de la ley, la rendición de cuentas, la tolerancia y el respeto por las minorías, etc.

Apunta Helio Gallardo que la democracia no existe, excepto como concepto-valor, en formas discursivas o discursos, que surgen en contextos determinados, alcanzando incidencia en las fuerzas sociales y en las instituciones. Puntualmente expone:

"Si la democracia no existe sino al interior de un discurso determinado ¿Qué existe entonces en la existencia diaria y material y no puramente como discurso? Lo que existe -responde el mismo Gallardo- son instituciones democráticas que condensan y expresan, mejor o peor, lógicas democráticas". Estas instituciones son el resultado: "del juego de diversas fuerzas sociales en el marco de un Estado de Derecho (...), o sea, de una convivencia regulada y sancionada por leyes de aplicación universal (general), y que funcionan de un modo tal que nadie puede ser perseguido y hostilizado, excepto por la comisión o sospecha de comisión de algún delito. En este Estado, nadie, tampoco, es un ciudadano por encima de toda sospecha. Lo que vale para los individuos vale asimismo para los sectores sociales. A este decantamiento de instituciones que funcionan de acuerdo a una lógica democrática lo englobamos bajo la expresión o concepto procesos de democratización". ${ }^{16}$

Al decir de Gallardo, los procesos de democratización no se concentran necesariamente en instituciones reductivamente políticas (como sería el caso

\footnotetext{
${ }^{16}$ Gallardo, Helio, Democratización y Democracia en América Latina, edición de la Facultad de Derecho de la Universidad Autónoma de San Luis Potosí, México, 2007, p. 153.
} 
de las oficinas del Ombudsman), sino que pueden materializarse igualmente en instituciones de la sociabilidad fundamental: la familia, el trabajo, la iglesia, etc. Para que esto suceda, tales instituciones deben organizarse con base en una lógica democrática. ¿En qué consiste esa lógica democrática? Así responde a la pregunta Gallardo: ${ }^{17}$

"Modernamente, democrático se opone a autocrático, es decir a Estados y gobiernos cuyo poder se gesta y reproduce por sí mismo, como el poder del padre en la familia patriarcal o el del amo sobre el esclavo. El poder autocrático despótico exige súbditos obedientes e incapaces de gobernarse por sí mismos, ya se trate de hijos, mujeres, esclavos o ancianos. Democracia hace por ello referencia a un poder no natural, gestado no por súbditos sino por ciudadanos activos que se interesan por la cosa común o pública (república) y participan en su administración y control. Un ciudadano posee, por tanto, autonomía (que se deriva y materializa de su información y capacidad para actuar), consigue autoestima con su participación en la vida pública, e irradia esta autoestima (colectiva) a otros. Por tanto las instituciones con lógica democrática se dan condiciones (gestación, organización) que potencian la autonomía, la autoestima y la irradiación o testimonio de la cosa pública en el ciudadano y, como debería ser obvio, en el ser humano como experiencia genérica, o sea universal (énfasis añadido).

Utilizando los argumentos de Helio Gallardo, transportándolos al Defensor del Pueblo en tanto institución con lógica democrática, resalta su característica de autonomía -principio básico de su actuación-, el desarrollo de su autoestima, que deriva necesariamente de su oportuna y constante presencia en los asuntos de interés social general, de donde deriva la adhesión de la sociedad en su conjunto a los principios del respeto, tolerancia y no discriminación, en tanto ejes rectores del actuar defensorial.

Las diversas opciones teóricas que explican la democracia, su contenido material y sus verdaderos alcances, es algo que queda fuera de nuestra consideración en esta ocasión. Interesa, en cualquier caso, dejar clara la vinculación que advertimos entre el quehacer del Defensor del Pueblo y la democracia.

El Defensor del Pueblo, apelativo de tanta majestuosidad, abre enormes expectativas en los diversos colectivos sociales. Igual no puede resolverlo todo, pero al menos debe intentar construir las bases de una vida democrática respetuosa, incluyente y solidaria. Para emprender tan compleja

${ }^{17}$ Gallardo, op. cit. p. 154. 
empresa, tal vez sea bueno recordar la definición de democracia que nos legó María Zambrano al decir que ésta, la democracia, es "la sociedad en la cual no sólo es permitido, sino exigido el ser persona" ${ }^{18}$

Del Defensor del Pueblo podemos esperar muchas cosas, pero no tanto como esperamos esta: que haga todo lo posible por garantizar que nuestras sociedades no impidan a nadie ser persona.

Finalmente, retomo aquí un interesante y provocador texto, que igualmente armoniza (por lo polémico) con el tema de éste artículo. Se trata de un documento suscrito por Fernando Mires, ${ }^{19}$ Catedrático de la Universidad de Oldenburg, Alemania, y que consigna siete mandamientos (reduccionistas, ciertamente, aunque también incitadores) ${ }^{20}$ sobre la democracia en oposición a la dictadura. Aquí los mandamientos:

I. La tolerancia política ha de terminar justo allí donde comienza una dictadura.

II. La peor de las democracias es mil veces preferible a la mejor de las dictaduras.

III. Nunca justifiques ni defiendas una dictadura para los demás si tú mismo (a) no quisieras vivir bajo ella.

IV. Nunca defiendas a una dictadura porque sea de la izquierda, del centro o de la derecha. Toda dictadura significa la abolición de la izquierda, del centro y de la derecha.

V. En un conflicto entre una nación democrática y una dictadura nunca tomes partido a favor de una dictadura. Eso siempre se paga muy caro.

VI. Nunca justifiques una dictadura por algunos de sus "éxitos" económicos o sociales. Recuerda siempre que Hitler terminó con la desocupación en Alemania, que Stalin industrializó la URSS en pocos años y que Pinochet detuvo la inflación en Chile.

\footnotetext{
${ }^{18}$ En Persona y Democracia, capítulo V.

${ }^{19}$ Originalmente, estos mandamientos se publicaron en la Revista América Latina en Movimiento, número 389, octubre de 2004 en Quito, Ecuador y son reproducidos por Helio Gallardo en la obra suya que hemos citado en este texto.

20 Helio Gallardo, op. cit., formula serias críticas a los siete mandamientos sobre la Democracia de Fernando Mires; con independencia de las coincidencias encontradas con los argumentos críticos de Gallardo, nada impide que el heptálogo cumpla, al menos, un propósito específico: alentar la polémica y la discusión, lo que efectivamente se logró en el ánimo de Gallardo.
} 
VII. La frase de Churchill: "la democracia es la peor forma de gobierno, con excepción de las demás" no es sólo un juego de palabras. "La peor" significa que no es perfecta, es decir, siempre es mejorable. "De todas las demás" significa que es la mejor hasta ahora posible.

El texto, crítico, escrito por Helio Gallardo donde analiza pormenorizadamente estos mandamientos no tiene desperdicio y conviene leerlo detenidamente. Con prudencia, pienso que para los objetivos de estas reflexiones, la pura enunciación de estos mandamientos resulta al menos inquietante y mueve a la reflexión. Creo que, en un primer momento plantea un desiderátum ético, político y jurídico: la defensa de los derechos humanos no es una tarea neutra, es una opción por la democracia; en tal caso, las Defensorías del Pueblo sí son instrumentos idóneos para el fortalecimiento de la vida democrática. 
